\title{
Aportes del Proyecto de Educación Rural (PER)
}

\section{Contributions of the Rural Education Project (PER)}

\author{
Kenneth Alfredo Cubillo Jiménez ${ }^{1}$ \\ División de Educación Rural \\ Centro de Investigación y Docencia en Educación \\ Universidad Nacional \\ Heredia, Costa Rica \\ kecuji@gmail.com \\ Urania María Chaves Murillo ${ }^{2}$ \\ División de Educación Rural \\ Centro de Investigación y Docencia en Educación \\ Universidad Nacional \\ Heredia, Costa Rica \\ uraniacm@gmail.com
}

Recibido 05 de mayo de 2010 • Aceptado 31 de agosto de 2010

Resumen.El presente artículo se deriva de la investigación "El proceso de formación inicial del Proyecto de Educación Rural (PER)", desarrollada entre 1984 y 1987 en las Direcciones Regionales de Educación de San Carlos, Coto y Limón, y sus aportes para los procesos formativos en la actualidad. El PER fue implementado por el Centro de Investigación en Docencia y Educación (CIDE), de la Universidad Nacional (UNA) y estaba orientado a la formación de educadores para escuelas unidocentes ubicadas en el contexto rural. El objetivo de la investigación se abocó a rescatar los aportes del PER a la luz de los procesos formativos que se requieren en la actualidad, dado que el éxito del PER tuvo como eje fundamental el liderazgo de los docentes para potenciar el desarrollo comunitario del contexto rural desde el aula, insumo por considerar en los nuevos procesos formativos en la educación rural.

Palabras claves. Contexto rural, educación rural, escuela unidocente, procesos formativos.

\footnotetext{
Académico, investigador y extensionista de la División de Educación Rural del Centro de Investigación y Docencia en Educación de la Universidad Nacional, Costa Rica. Académico (tutor virtual) del Centro Regional de Cooperación Regional para la Educación de Adultos de América Latina y el Caribe (CREFAL). Experiencia docente en primaria, secundaria y educación superior. Educador en entornos virtuales. Magíster en Formación de Formadores de Docentes de Educación Primaria. Licenciado en Educación con énfasis en Educación Rural, I y II ciclos.

2 Académica de la División de Educación Rural del Centro de Investigación y Docencia en Educación de la Universidad Nacional. Experiencia docente en primaria, secundaria y educación superior. Maestría en Formación de Formadores de Docentes de Educación Primaria. Máster en Ciencias de la Educación con énfasis en Administración Educativa. Máster en Ciencias de la Educación con énfasis en Currículo.

3 El lenguaje que se utiliza en este informe es incluyente en materia de género.
} 


\begin{abstract}
This paper presents the results of the research project "El proceso de formación inicial del Proyecto de Educación Rural (PER)" (creation of the Rural Education Project, PER by its Spanish acronym), conducted between 1984 and 1987 in the Regional Offices of the Ministry of Public Education in San Carlos, Coto and Limón. The Rural Education Project (PER) was implemented by the Center of Research and Teaching in Education (CIDE) of the Universidad Nacional (UNA) aiming at training teachers of single-teacher schools located in rural areas. The objective of our research was to collect the contributions of PER bearing in mind the training processes required today, and considering the success of PER, which was based on the leadership of teachers to promote community progress in rural areas from the classroom, an input to be considered in the new learning processes of Rural Education
\end{abstract}

Keywords. Rural context, rural education, single-teacher school, training processes.

\title{
Introducción
}

Este artículo presenta los resultados de la investigación "Aportes del Proyecto de Educación Rural", en adelante conocido como el PER, a partir de su génesis e implementación, y de los procesos formativos que se requieren en la actualidad.

El PER se basó en el proceso de formación inicial de educadores para escuelas unidocentes ubicadas en el contexto rural. La Universidad Nacional lo desarrolló entre 1984 y 1987, en las Direcciones Regionales de Educación de San Carlos, Limón y Coto.

El análisis del trabajo se estructuró en dos apartados: Generalidades del PER y Principales hallazgos del PER.

Antes de desarrollar cada uno de estos apartados, se aclaran los conceptos: contexto rural, educación rural y escuela unidocente.

\section{El contexto rural}

En primera instancia, según Van Kampen y Ovares (2003), citados por Angulo et al. (2008), es posible caracterizar el contexto rural desde la realidad de las comunidades como:

- Un espacio geográfico que experimenta un desarrollo urbano a partir de las migraciones de personas de la ciudad al campo y del campo a la ciudad.

- Su economía se ha diversificado al cambiar el uso de sus tierras. En el pasado se dedicaban a la agricultura y a la ganadería. En la actualidad, a otros productos no tradicionales, con ayuda de la tecnología; al turismo ecológico, al turismo campesino, al comercio y a otras actividades informales.

- Su forma de producir incrementa los círculos de pobreza y la exclusión social.

- La existencia de buenas prácticas relacionadas con la conservación del medio ambiente para fomentar el turismo, aunque con persistencia de problemas relacionados con el manejo de los desechos. 
- Su identidad cultural se ha transformado por la influencia de los medios de comunicación, existen nuevos valores.

- Surgimiento de la mujer en su escenario económico al ser jefe de hogar y tener la responsabilidad de la familia, lo cual la obliga a salir de su casa e incorporarse a las labores remuneradas.

- Un ámbito educativo donde la educación no formal juega un papel importante, sin embargo, aún no ha sido aprovechado en los procesos de desarrollo comunal.

- Existencia, en su mayoría, de escuelas unidocentes, ubicadas en territorios o comunidades muy distintas, desde las que tienen acceso a vías de comunicación y medios de transporte, hasta las que carecen de estos (como las de las zonas indígenas, por lo que sus procesos de aula difieren de los de la ciudad).

Según lo anterior, se puede afirmar que el contexto rural está conformado por comunidades ubicadas en un espacio geográfico donde se ha diversificado la economía, la identidad cultural se ha transformado por la influencia de los medios de comunicación, existen buenas prácticas desde la educación no formal, la mujer juega un papel importante en el nivel económico y la mayoría de sus escuelas son unidocentes.

Por otro lado, para Rodríguez (2008) el contexto rural:

(...) se construye sobre la base de espacios geográficos compartidos, de historias vividas y entrelazadas, de orígenes étnicos, con tradiciones, expresiones organizacionales y sociales, relaciones entre las personas y con el entorno, y formas de producción y productos que en conjunto contienen identidades, culturas, saberes, prácticas y valoraciones (...) (p. 13)

En este caso, Rodríguez enfatiza en varios elementos que constituyen el contexto rural: el espacio geográfico, la historia, la etnia y las relaciones que conforman su cultura a partir de sus conocimientos, experiencias y forma de valorar el mundo.

Al comparar los elementos de las definiciones expuestas por Angulo et al. (2008) y Rodríguez (2008), se puede concluir que el contexto rural es el espacio geográfico donde conviven personas con orígenes étnicos en común; con un patrón cultural compartido; con conocimientos, experiencias y formas de valorar el mundo desde la educación no formal y, en algunas ocasiones, desde la educación formal o educación rural.

\section{La educación rural y la escuela unidocente}

La educación en el contexto rural, en adelante, "educación rural", se entiende como un espacio para el aprendizaje, y trasciende el recuento de las prácticas educativas -para la aplicación del currículo nacional- que se desarrollan en las instituciones de educación ubicadas en el contexto rural del país: implica la construcción permanente de una pedagogía particular desde el contexto educativo rural y en este, donde el docente juega un papel fundamental porque es el encargado de facilitar y liderar los procesos educativos, tanto dentro como fuera del aula. 
Así, la educación rural podría definirse como la educación que se imparte en (o desde) centros públicos o privados de preescolar, de I y II Ciclos (primaria), de III Ciclo y de Ciclo Diversificado (académico o técnico, secundaria), así como de enseñanza especial y los proyectos curriculares alternativos ubicados en espacios geográficos que el Instituto Nacional de Estadística y Censo [INEC] (2004), define como "rural concentrado y rural disperso".

La mayoría de las instituciones de primaria ubicadas en el contexto rural son denominadas escuelas unidocentes.

En Costa Rica, administrativamente, se consideran escuelas unidocentes aquellos centros educativos de I y II ciclos cuya matrícula inicial no sobrepasa los 50 estudiantes. Esta modalidad surge en Costa Rica en la década de los sesenta, como una alternativa que les permitiera, a las zonas rurales, contar con oportunidades educativas que, dadas la lejanía y dificultad de acceso, de otra forma no podrían brindarse.

Desde entonces se han implementado diversas estrategias con el fin de ampliar la cobertura y posibilitar que, en los rincones más escondidos del territorio nacional, se cuente con una escuela unidocente que ofrezca la educación primaria completa.

En la actualidad, las escuelas rurales están distribuidas a lo largo de todo el territorio nacional y representan aproximadamente el $70 \%$ de las instituciones de I y II ciclos del país.

Según los datos del Ministerio de Educación Pública del 2007, existen en Costa Rica 1.943 escuelas unidocentes con menos de 51 estudiantes matriculados al inicio del curso.

Una situación que ha afectado la educación rural, específicamente las escuelas unidocentes, es la necesidad de contar con educadores formados para que respondan a una sociedad cambiante. El educador se enfrenta a una creciente demanda social para el desarrollo de habilidades de aprendizaje de los estudiantes, por lo que se le exige que no solo adquiera conocimientos, sino que sea capaz de formar parte del desarrollo comunitario.

En la década de los años 80, Costa Rica enfrentó una dura crisis respecto a la escasa oferta de docentes certificados por las universidades, por lo que, en algunas escuelas unidocentes, los maestros han ejercido sin estar titulados, en calidad de aspirantes. Un aspirante es aquel docente que carece de formación universitaria y que desconoce aspectos pedagógicos para la atención de niños y para la administración de un centro educativo.

El PER nace en la década de los 80 , precisamente, para responder a la creciente necesidad de maestros debidamente capacitados.

\section{Generalidades del PER}

El PER nació en 1983 con el nombre de "Proyecto experimental de formación de educadores con concentración en la problemática del área rural”, como respuesta a la falta de maestros titulados para zonas rurales. Según Nieva (s. f.), “(...) el docente generalmente proviene de ámbitos urbanos con una “cosmovisión' creada por su propio universo simbólico (...)” (p. 7). Es decir, los docentes tienen un concepto de desarrollo y progreso relacionado con su realidad y esta generalmente tiene como referencia el mundo urbano. Amttman (2000), citado por Parra (s. f), reitera esta posición al afirmar que "(...) los profesionales presentan debilidades que se traducen en desconocimiento de la 
complejidad rural, segmentación disciplinaria en su análisis, desvalorización de las formas de vida en el campo y desconocimiento de modelos de intervención en las distintas comunidades rurales" (p. 3).

En concordancia con lo anterior, la Universidad Nacional (UNA) y el Ministerio de Educación Pública (MEP) firmaron, en 1983, un convenio para la formación de docentes que atendían las escuelas de las zonas rurales. Según el Centro de Investigación y Docencia [CIDE] (1986), el objetivo del convenio era formar docentes para que fueran capaces de incorporar e incorporarse a las comunidades en los procesos de aula, con el propósito de comprender la realidad comunal y contribuir a su transformación, a partir de conocimientos metodológicos, pedagógicos y didácticos, necesarios para el logro del desarrollo comunal.

Para realizar el trabajo estipulado en el convenio, se creó una comisión interinstitucional (UNA-MEP) encargada de organizar la primera etapa del trabajo en tres vías: diagnosticar la realidad rural, seleccionar a los estudiantes y profesores, y diseñar el plan de estudio.

El trabajo se inició con una exploración en tres regiones del país con una situación crítica en cuanto a disponibilidad de maestros. Se pretendía identificar aspectos propios y percepciones de la escuela y de los habitantes de las comunidades rurales, el tipo de maestro que la comunidad requería y la forma en que debería desempeñarse un docente rural.

Según CIDE (1986), en los resultados de esta exploración prevalecieron las escuelas unidocentes, atendidas por maestros aspirantes. En ese entonces, un 35\% de los docentes de todo el país eran aspirantes, problemática que incidía directamente en la disposición, metodología, recursos didácticos y confianza para el desarrollo del proceso de aprendizaje en el aula.

Durante el diagnóstico, la UNA se encargó de visitar comunidades rurales para realizar las entrevistas a informantes claves de las comunidades y de sus escuelas. El MEP, por otro lado, se comprometió a nombrar como docentes en escuelas rurales a los estudiantes del PER, y a darles el permiso de estudio para que asistieran los viernes, cada dos meses, a recibir lecciones en los centros de población de las regiones. En ese entonces, se seleccionan como regiones prioritarias San Carlos, Coto y Limón, por la gran cantidad de maestros aspirantes sin ninguna formación docente que había en estas zonas.

Durante el desarrollo del proyecto, con la incorporación de todas las actividades prácticas que integraban diferentes asignaturas desde la planificación, la práctica profesional y la evaluación, a fin de responder a los intereses y necesidades de una comunidad, se les ofrecieron, a los estudiantes que estaban laborando como unidocentes, las herramientas para que integraran los componentes teóricos y prácticos de su formación, en los diferentes momentos de su proceso formativo. En fin, el PER pretendía “(...) desarrollar una actitud y un proceso metodológico que le permita al educando de este plan partir de su realidad y reflexionar críticamente sobre su práctica” (CIDE, 1986, p. 16). Para lograr lo anterior, se trabajó en la elaboración de un plan de estudios acorde con las demandas de la sociedad de esa época.

El plan de estudios se basó en cuatro componentes curriculares: salud, recreación, desarrollo comunal y trabajo de aula. De acuerdo con esta concepción, el desarrollo del proyecto se conceptualizó como un proceso de trabajo en equipo, que planificó y buscó alternativas y se comprometió con el desarrollo comunitario operacionalizando los objetivos, por lo tanto, realizó una planificación realista, implementó las propuestas, las revisó constantemente y 
las adaptó a las nuevas situaciones y a las demandas de los estudiantes y del entorno. Este proyecto se caracterizó por su enfoque metodológico dinámico, participativo y flexible, donde prevaleció el planteamiento de la acción-reflexión, que buscaba, desde la práctica, un conocimiento y mejoramiento de la realidad. De acuerdo con CIDE (1986), este enfoque de trabajo garantiza la “(...) flexibilidad, participación y mayor concordancia con los intereses y necesidades planteadas por los estudiantes" (p. 34).

Otro aspecto relevante de este proyecto fue la forma en que se trabajaban las disciplinas: conjuntamente en torno a proyectos de trabajo multidisciplinares o núcleos generadores, donde se correlacionaban las asignaturas del área conceptual y del área operativa con el área problema. Cabe recordar que el área problema se refirió a la integración del currículo escolar con las necesidades y expectativas de la comunidad y viceversa, y que las demás asignaturas debieron, constantemente, realimentar esta integración (CIDE, 1986).

Este plan de estudio fomentaba los procesos de construcción social para obtener y utilizar nuevos conocimientos, destrezas, conductas y valores, fomentando nuevos métodos de trabajo y saberes específicos, e incrementando las expectativas de supervivencia y desarrollo de la organización por lo que surge un plan de estudios:

(...) de tipo nuclear correlacionado, en el cual los cursos trabajan independientemente; pero todos incorporan parte de su tiempo a la correlación; es decir a aportar elementos conceptuales y operativos para que se dé la integración de la temática comunal en el currículo oficial (CIDE, 1986, p. 29).

En fin, se buscaba formar un educador para que se integrara a poblaciones con una compleja situación socio-económica y cultural, por lo que se requería una orientación que transformara el clásico plan por disciplinas, en el cual el docente transmitía los conocimientos encajonados en forma aislada, en lugar de brindar una formación integral donde se plasmara la relación entre las diferentes áreas de la vivencia humana.

Es decir, el plan buscaba la formación de docentes para fortalecer la relación comunidadescuela y escuela-comunidad, principios fundamentales del PER, la vinculación entre la formación, la investigación, el servicio a la comunidad y la búsqueda de alternativas de desarrollo integral en las zonas rurales.

El plan de estudios se implementó por medio de la modalidad presencial y a distancia.

Según CIDE (1986), la modalidad presencial consiste en el "(...) intercambio directo de experiencias entre profesores y alumnos (...)" (p. 34). En el caso del PER, dicha modalidad se implementó cuando los docentes encargados de los cursos visitaban los núcleos de trabajo del PER (Zona Norte, Ciudad Neilly y Siquirres). Este encuentro presencial buscó el intercambio y análisis de experiencias, facilitó la entrega de documentos a los estudiantes para su lectura y estudio, permitió la evaluación de aprendizajes adquiridos y fomentó el conocimiento de procedimientos adecuados para diversas situaciones que los estudiantes plantearan.

En cuanto a la modalidad a distancia, se concibió como el espacio de aprendizaje donde los estudiantes, sin estar en contacto con los docentes formadores, trabajaban cumpliendo con una serie de obligaciones. El trabajo a distancia se concibió como la base para el desarrollo de 
la clase presencial. En este espacio, los estudiantes del PER presentaron los resultados del trabajo implementado. Las siguientes son ejemplos de actividades en la modalidad a distancia:

- Lecturas dirigidas

- Ejecución de proyectos docentes y comunitarios.

- Realización de esquemas, resúmenes, análisis de casos.

\section{Principales hallazgos del PER}

Para obtener los resultados de este trabajo de investigación, se trianguló la información obtenida mediante la implementación de tres grupos focales y de un cuestionario a los graduandos del PER. Además, algunos de los profesores del PER participaron en una entrevista. Tanto los tres grupos focales, como la entrevista y el cuestionario se orientaron por tres tópicos que respondían a los objetivos específicos:

1. Identificar fortalezas y debilidades del PER desde la óptica de algunos profesores y egresados que participaron en el proyecto. Egresados (salud, agricultura, recreación, trabajo aula, comunidad). Profesores en general.

2. Establecer qué elementos del PER podrían servir de base para un nuevo plan de formación inicial de educadores para zonas rurales.

3. Determinar qué influencia tuvo la formación del PER en el desarrollo de las comunidades, según la percepción de los participantes.

Por lo que, en concordancia con los objetivos específicos, el análisis del PER como trabajo de investigación se fundamentó en los siguientes tópicos:

1. Fortalezas y debilidades del PER

2. Elementos del PER como base para un nuevo plan de formación inicial de educadores unidocentes para zonas rurales.

3. Aportes del PER en el desarrollo de las comunidades rurales.

Los siguientes resultados, según los tópicos antes mencionados, derivan de lo expresado por los graduados y profesores, a partir de los grupos focales de la investigación:

\section{Fortalezas y debilidades del PER}

\section{Fortalezas}

- El proceso de selección de estudiantes utilizó estrategias como pruebas de aptitud y actitud, así como entrevistas para identificar la vocación de los aspirantes que deseaban laborar en escuelas unidocentes de contexto rural. 
- El plan de estudios se fundamentó en los componentes curriculares: trabajo de aula, recreación, salud y desarrollo comunal. La formación docente se visualizó como un acto integral que trasciende el aula escolar.

- La formación inicial incorporó actividades extracurriculares como campamentos, visitas guiadas a sitios de interés cultural, talleres, conferencias, seminarios, foros, encuentros regionales para el fortalecimiento integral de los estudiantes en las áreas: cultural, ética, histórica, geográfica y estética.

- El seguimiento al estudiantado permitió comprobar la relación entre la teoría (recibida en la universidad) y la práctica (aplicabilidad en el aula escolar y en la comunidad rural).

- Las experiencias de aprendizaje contextualizadas evidenciaron que los profesores formadores conocían la realidad de los estudiantes y de las comunidades rurales, lo que les permitía fortalecer la formación personal.

\section{Debilidades}

Las debilidades que señalaron las participantes y los participantes están relacionadas con los aspectos emocional y familiar (soledad al vivir lejos del hogar), económico (falta de pago oportuno) y académico (lejanía con el centro de estudio).

\section{Elementos del PER como base para un nuevo plan de formación inicial de educadores para zonas rurales}

- Contemplar actividades extracurriculares que enriquezcan la formación del docente en las áreas cultural, ética, histórica, geográfica y estética.

- Considerar la formación en valores, las actitudes, las aptitudes y los conocimientos que requiere el trabajo con niños, padres de familia y comunidades en zonas rurales.

- Brindar seguimiento a los estudiantes durante el proceso de formación y no limitarse únicamente a la práctica supervisada. La educación como proceso continuo, permanente, de crecimiento personal y profesional del estudiante, implica un compromiso más amplio, que trasciende el aula universitaria. Es hacer vida la relación acción-reflexión-acción.

- Contextualizar la mediación pedagógica para que resulte pertinente y significativa. Se hace necesario que la formación docente sea más práctica, cercana a la realidad y que implique aplicabilidad y funcionalidad en la persona que se forma.

- Contemplar la participación y la horizontalidad como elementos esenciales en la formación inicial. Es un proceso de formación de adultos que requiere respeto, construcción conjunta del conocimiento y toma de decisiones en equipo.

\section{Aportes del PER en el desarrollo de las comunidades rurales}

- La creación de proyectos a partir del trabajo con los núcleos generadores desde el aula, permitió el acercamiento de los padres de familia y la comunidad a la escuela. 
- Proyectos de infraestructura escolar, como la construcción y mejoramiento del aula, el comedor, los baños y el ornato de las instituciones.

- La participación activa y comprometida de los comités de apoyo (Junta de Educación y el Patronato Escolar), en el desarrollo de los proyectos.

- La integración del docente, como líder, en el trabajo comunitario (construcción de caminos, salones comunales, acueductos y hasta la formación de cooperativas rurales).

- Una relación excelente del docente con los miembros de la comunidad donde destaca el diálogo, la cooperación, el respeto y una actitud de "puertas abiertas".

- El favorecimiento de una comunicación horizontal con los padres de familia, mediante las visitas periódicas que se realizaban a los hogares.

A partir del análisis anterior, los planes de estudio, actuales y futuros, para la formación de maestros de zonas rurales, deben tomar en cuenta los siguientes aspectos:

- Contar con componentes curriculares que respondan a las demandas de la sociedad actual como: trabajo de aula, tecnologías de la información y la comunicación (TIC), recreación, arte, música, salud, ambiente y desarrollo comunitario.

- Incorporar una gran cantidad de actividades extracurriculares que enriquezcan la formación del docente en las siguientes áreas: cultural, ética, histórica, geográfica, estética, entre otras. Esto significa realizar actividades tales como campamentos, visitas guiadas a sitios de interés cultural, talleres, conferencias, seminarios, foros y encuentros regionales.

- Considerar en el perfil del formador de formadores los valores (solidaridad, compañerismo, responsabilidad, valentía, honestidad, compromiso, humildad, creatividad, tolerancia, perseverancia, dedicación, y el respeto entre otros), las actitudes, las aptitudes y los conocimientos que requiere el trabajo con niños, padres de familia y comunidades en zonas rurales.

- Visitar a todos los estudiantes para darles seguimiento durante todo el proceso de formación y no solamente como requisito de práctica supervisada.

- Trabajar la motivación de los estudiantes (creación de afectos, comprensión de la realidad, vivencia y experiencias).

- Favorecer el desarrollo de la creatividad como elemento indispensable en cada educador que se forme.

- Propiciar que los profesores conozcan la realidad de las comunidades rurales, favorecer experiencias de aprendizaje contextualizadas; desarrollar en sus estudiantes comportamientos como el amor por el trabajo, creer en lo que se hace y enseñar con el ejemplo.

- Fomentar la importancia de la educación como vehículo de desarrollo comunal.

Ahora bien, hay que tomar en cuenta que el PER surgió en un período con especificidades históricas que lo hicieron posible; el país presentaba un importante faltante de docentes para las zonas rurales y era urgente que se formaran. La realidad histórica actual presenta un panorama completamente diferente, dada la cantidad de profesionales de la educación que ofertan sus servicios para trabajar en las instituciones educativas costarricenses.

La necesidad de hoy plantea nuevos retos: actualizar y capacitar al docente en servicio, en temas relacionados con la ruralidad, para permitirle a este profesional de la educación, un 
remozamiento de los conocimientos obtenidos en la vida académica, lo cual favorece la calidad de la educación en dichos contextos.

\section{Referencias bibliográficas}

Angulo, L., Carvajal, V., Cerdas, Y., Méndez, N., Morera, D.,Torres, N. y Van Kampen, P. (2008). Formación docente para la educación básica: Énfasis en la ruralidad en Centroamérica y República Dominicana. San José, Costa Rica: URUK Editores.

Centro de Investigación y Docencia en Educación [CIDE]. (1986). Plan de estudios para el Proyecto experimental de formación de educadores con concentración en la problemática del área rural. Heredia, Costa Rica: Autor.

Instituto Nacional de Estadística y Censo [INEC]. (2004). Documento metodológico. V Censo Nacional de Vivienda del año 2000. [Área de Censos y Encuestas]. Recuperado de http:// www.inec.go.cr/A/MI/Publicaciones/SerieMetodológica/Metodologías/07.Metodología Censo Vivienda.pdf

Nieva, F. (s. f). La formación de grado inicial y contextos de ruralidad en el marco de la transformación educativa: El profesorado en geografía para la EGB 3 y Polimodal del IES Monteros-Tucumán. Buenos Aires, Argentina: Instituto de Enseñanza Superior Monteros. Recuperado de http://iesm.tuc.infd.edu.ar/sitio/print.cgi?wid seccion=6\&wid $\underline{\text { item }=\& w O u t=\text { topdf }}$

Parra, J. E. (s. f.). Educación para una nueva ruralidad. Recuperado de http://www.misionrural.

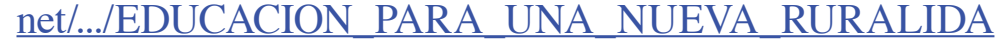

Rodríguez, E. (2008). Currículo para la escuela rural en un proceso de cambio cultural. Revista Centroamericana de Educación, 3(1), 95-116. 